\title{
Intramammary Sentinel Lymph Nodes: What is the Clinical Significance?
}

\author{
Charles E. Cox, MD, John M. Cox, MD, Daniel Ramos, BS, and Tammi L. Meade, BS
}

Department of Surgery at the Comprehensive Breast Cancer Program, H. Lee Moffitt Cancer Center and Research Institute at the University of South Florida, Tampa, Florida, USA

Sentinel lymph node biopsy (SLNB) has been widely accepted and used to determine axillary lymph node status for the past decade. ${ }^{1,2}$ Consequently, SLNB has been a valuable method for determining patients' treatment options, such as whether or not a complete axillary lymph node dissection (CALND) would be necessary for local control and correct staging of the breast cancer patient. When extraordinary cases materialize, it is often difficult to determine a common treatment pathway for the patient. The rare finding and subsequent treatment of the intramammary sentinel lymph node (IntraMSLN) metastasis has become such in recent years. The argument lies in whether or not a positive IntraMSLN warrants a CALND or whether it can be spared, compared with a positive axillary sentinel lymph node, which would warrant a CALND.

Two studies have examined the prognostic significance of positive intramammary lymph nodes (IntraMLN) from the presentinel node era and postulated treatment recommendations. Shen et al. reported that IntraMLN metastasis was an independent predictor of a worsened prognosis and that patients with IntraMLN metastases were significantly associated with a shorter disease-free survival and overall survival. Therefore, CALND was warranted. ${ }^{3}$ Similarly, Guth et al. reported that a CALND should be recommended for patients with positive IntraMLNs based on their findings that patients with IntraMLNs had more aggressive cancers including higher rates of lymphovascular invasion and axillary

Published online March 6, 2008.

Address correspondence and reprint requests to: Charles E. Cox, MD; E-mail: coxce@moffitt.usf.edu

Published by Springer Science+Business Media, LLC $\odot 2008$ The Society of Surgical Oncology, Inc. nodal disease, and these patients were more frequently multifocal and had a higher grade and stage of disease on final analysis. ${ }^{4}$

In contrast to previous studies reported in which IntraMLNs were detected on final pathology review, Dr. Intra and colleagues recently reviewed their institution's data for patients with IntraMSLNs detected with mapping techniques. Twenty-two patients were found to have IntraMSLN and axillary sentinel lymph nodes (axSLN) present. Of the 22 patients, 15 had both IntraMSLNs and axSLNs excised, of which six IntraMSLNs were positive and all axSLNs were negative. In addition, two of the six patients had positive IntraMSLNs, and one patient with a negative axSLN had a CALND; in all three cases all axillary nodes were negative. After 24 months they found no recurrences. Based on these findings, Dr. Intra's group argued that when both IntraMSLNs and axSLNs are present then both should be biopsied and that a CALND should be based on the status of the axSLNs. ${ }^{5}$ Dr. Intra and colleagues raise the question whether a CALND on patients should be done when positive or negative IntraMSLNs are found when axSLNs are not localized. The answer to which this author's recommendation would be, yes perform the CALND due to mapping failure of the axilla.

Between September 1994 and October 2005, 15,000 patients' records in the Moffitt Cancer Center Breast Cancer database were retrospectively reviewed following institutional review board (IRB) approval. Only patients with T1-T2 invasive breast tumors were included. Ninety-one patients were identified with having IntraMLNs, of which eight patients (9\%) had isolated IntraMLNs. All eight patients with isolated intraMLNs had negative axillary contents on pathology. Eighteen of the 91 patients $(21 \%)$ had 
IntraMLN with axillary metastases. Sixty-two of 91 patients $(69 \%)$ had a negative IntraMLN. There were 502 patients identified with (N1), axillary nodal metastasis and compared with patients with isolated IntraMLN positive patients. Patients with isolated IntraMLN metastases had 0 of 8 recurrences and 0 of 8 deaths after an average follow-up time of 2.63 years. Patients with solitary axillary metastasis had 36 to 502 recurrence $(7 \%)$ and 44 of $502(9 \%)$ died after an average of 2.0 years follow-up. When an isolated positive IntraMLN is found, these data suggest an improved prognosis over those patients with an isolated axillary SLN and would not be deemed a high-risk patient for metastasis to the remaining nodes in the axilla.

This Moffitt Cancer Center series of IntraMSLNs reported at the 2005 Society of Surgical Oncology conference demonstrated that a positive isolated IntraMSLN compared with an isolated positive axSLN, actually had an improved prognosis. These data would confirm the study and recommendation of
Intra et al. ${ }^{5}$ that a positive IntraMSLN with negative axSNL would not warrant CALND nor should it predict a worse prognosis than isolated axillary nodal disease.

\section{REFERENCES}

1. Lyman G, Giuliano A, Somerfield M, et al. American society of clinical oncology guideline recommendations for sentinel lymph node biopsy in early-stage breast cancer.. J Clin Oncol 2005; 23:7703-20.

2. Takei H, Kurosumi M, Yoshida T, et al. Current trends of Sentinel lymph node biopsy for breast cancer-A surgeon's perspective. Breast Cancer 2007; 14:362-70.

3. Shen J, Hunt KK, Mirza NX, et al. Intramammary lymph node metastases are an independent predictor of poor outcome in patients with breast carcinoma. Cancer 2004; 101:1330-7.

4. Guth AA, Mercado C, Roses DF, et al. Intramammary lymph nodes and breast cancer: a marker for disease severity, or just another lymph node? Am J Surg 2006; 192:502-5.

5. Intra M, Garcia-Etienne CA, Renne $G$, et al. When sentinel lymph node is intramammary. Ann Surg Oncol 2007; DOI: 10.1245/s10434-007-9726-1 [online Dec 6, 2007]. 